\title{
Photoinduction of spontaneous surface relief gratings on Azo DR1 Glass
}

\author{
Leila Mazaheri, ${ }^{1}$ Sanyasi Rao Bobbara, ${ }^{1}$ Olivier Lebel, ${ }^{2}$ And Jean-Michel \\ NUNZI ${ }^{1,3^{*}}$ \\ ${ }^{1}$ Department of Physics, Engineering Physics \& Astronomy, Queen's university, Kingston K7L-3N6, Ontario, Canada \\ ${ }^{2}$ Department of chemistry and chemical Engineering, Royal Military College of Canada, Kingston K7K-7B4, Ontario, Canada \\ ${ }^{3}$ Department of chemistry, Queen's university, Kingston K7L-3N6, Ontario, Canada \\ *Corresponding author: nunzijm@queensu.ca
}

Received XX Month XXXX; revised XX Month, XXXX; accepted XX Month XXXX; posted XX Month XXXX (Doc. ID XXXXX); published XX Month XXXX

\begin{abstract}
Surface relief gratings were spontaneously photoinduced from a collimated Nd:YAG laser beam at $532 \mathrm{~nm}$ on thin films of a Disperse Red 1- functionalized glass-forming compound. Pattern formation was studied by measuring the diffraction intensity of a He-Ne laser probe beam at $633 \mathrm{~nm}$ and by atomic force microscopy (AFM). The dependence of pattern formation on both irradiation time and intensity was studied. The gratings could be erased both optically and thermally. The orientation of the gratings is influenced by the polarization of the writing beam, and it is accompanied by strong diffraction of the incident light into the sample plane, thereby providing a way to couple and trap the light into the substrate. Interestingly, photobleaching upon prolonged irradiation yields transparent gratings and the process is partially reversible upon thermal erasure.
\end{abstract}

OCIS codes: (230.1950) Diffraction gratings; (190.5940) Self-action effects; (310.6860) Thin films, optical properties.

http://dx.doi.org/10.1364/OL.99.099999

Surface relief gratings (SRG) have become a crucial element for several applications in photonics including the coupling of light into and out of optical waveguides [1], wavelength filters [2], optical data storage $[3,4]$, optical nano imaging [5,6], and in photovoltaic cells as light harvesting structures [7]. A traditional way to fabricate such gratings is by using lithography/etching techniques, which requires complex and expensive equipment and multiple processing steps. In contrast, light-assisted patterning constitutes a simpler, flexible, time- and cost-efficient alternative. In this respect, azobenzene-functionalized materials have been the subject of many studies for the holographic fabrication of photonic structures due to their remarkable photo-responsive properties [3, 8]. Irradiation of thin films of materials functionalized with azobenzene chromophores with an interference pattern of polarized laser beams results in reversible and controlled deformations of the film surface along specific patterns (SRG) due to cis-trans isomerization and mass transport [9-11]. Another advantage of SRGs formed optically, as opposed to etched patterns, is that the structures can be erased optically or thermally, and rewritten reversibly [12].

A lesser-investigated fact is that surface relief structures can also be formed under single-beam exposure [13]. In this case, no intensity or polarization variation is needed and the interference pattern is self-organized, resulting in well-organized structures. For surfaces that support surface-guided waves, the periodicity of the structure depends on the wave guiding properties of the surface [14]. For materials which do not support surface waves, the periodicity is in the range of the writing beam wavelength, which implies that interferences of the primary beam with selfinduced diffraction at grazing incidence plays a significant role in structure formation [13]. In ordinary dielectrics, a high-power pulsed laser of few $\mathrm{J} /$ pulse is used to create irreversible structures called laser induced periodic surface structures (LIPSS) [15]. In azo films, exposure to a single and uniform beam with moderate power of few hundred $\mathrm{mW} / \mathrm{cm}^{2}$ results in reversible surface relief grating formation owing to light-induced isomerization and mass movement. The formation of so-called spontaneous SRGs (SSRGs) depends on various experimental parameters such as wavelength, polarization, incidence angle and power of the incident laser beam, as well as on the molecular structure [16-18].

Various explanations to the formation of SSRGs were proposed: SSRGs may originate from the interference of an incident laser beam with beams that are scattered by the sample [16]; the rheological properties of polymers, which are dependent on chain length and the nature of the polymer - azo binding may also impact SSRG growth and shape [19]. To gain a deeper understanding of SSRG formation, the detailed mechanism of the growth process must be determined. A single-molecule material allows mitigating the impact of polymer's rheology on SSRGs. Furthermore, small molecules yield a more homogeneous behavior, not only between 
different samples, but also within a given sample because all molecules are identical [20]. While most azobenzene chromophores crystallize easily, limiting their usefulness for thinfilm applications, it is possible to generate azobenzene derivatives that can be processed into high-quality amorphous thin films [2123].

In this work, single beam induced gratings in different directions were inscribed in one simple step, and their behavior was studied. Disperse Red 1 (DR1) was selected as the azo chromophore for the present study as it shows rapid SRG growth at the working wavelength. A DR1 derivative with a mexylaminotriazine group (Fig. 1) was the material used in this study [20] owing to its capacity to form extremely stable glassy phases, and a rate of SRG growth closely similar to that of DR1-functionalized polymers.

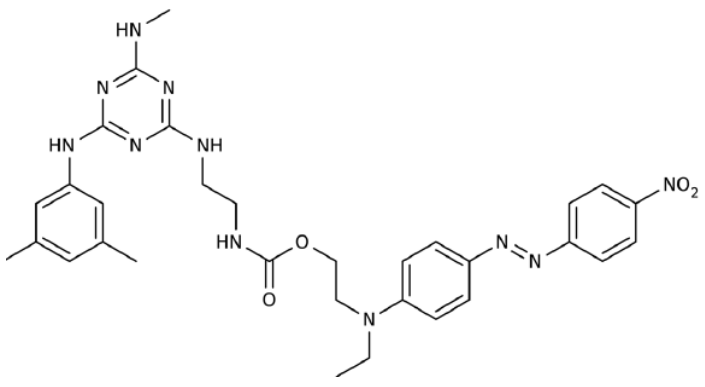

Fig. 1. The DR1 molecular-glass material used in this study.

SSRGs were inscribed on films of DR1-glass, and the dependence of SSRG growth rate and direction on writing beam intensity and polarization was studied. The presence of directional backscattered light was clearly observed for the first time, thereby showing evidence of the involvement of light coupling in the SSRG growth process, which was further confirmed by the lack of coherence in surface nanostructures formed in the absence of the incident beam.

DR1-glass was prepared according to a literature procedure [20]. Thin films of the material on glass substrates were spin-coated from a $30 \mathrm{mg} / \mathrm{mL} \mathrm{CH}_{2} \mathrm{Cl}_{2}$ solution. The solution was stirred for 1 hour and then filtered through a $0.45 \mu \mathrm{m}$ filter. The deposited thin films were then dried at $95{ }^{\circ} \mathrm{C}$ for 5 minutes to remove solvent traces. DR1-glass thickness was $270 \pm 30 \mathrm{~nm}$ as measured out of 10 samples.

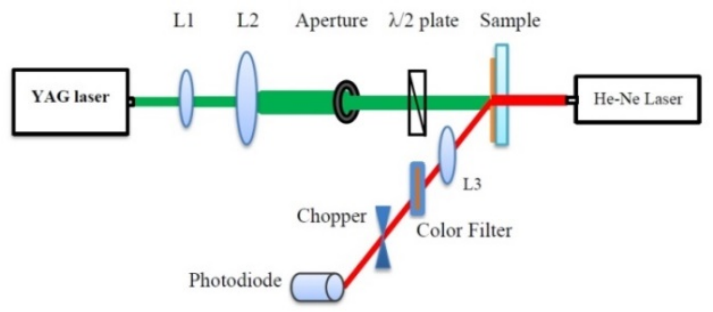

Fig. 2. Experimental setup: L1 and L2 are lenses to expand the collimated beam, and L3 is a lens to focus the diffracted beam on the detector.

A YAG laser at $\lambda=532 \mathrm{~nm}$ was used as pump beam. The size of the collimated beam impinging onto the sample was controlled with a Keplerian beam expander. The beam was set perpendicular to the sample and its polarization was set vertical. The polarization state can be tuned using half and quarter-wave plates. SSRG formation was monitored by measuring diffraction intensity of an $8 \mathrm{~mW} \mathrm{He}-$ Ne probe laser at $633 \mathrm{~nm}$ and by atomic force microscopy (AFM) in taping mode. The He-Ne laser was incident backwards on the spot as irradiated by the pump beam, where the SSRG forms, as illustrated in Fig. 2. The diffracted beam from the probe recorded at $9 \pm 3$ degrees was mechanically chopped and monitored by a silicon photo detector. The signal was detected by a lock-in amplifier, digitized by an oscilloscope and recorded by a computer. A colored filter placed in front of the detector blocked off selfdiffraction from the pump beam.

(a)
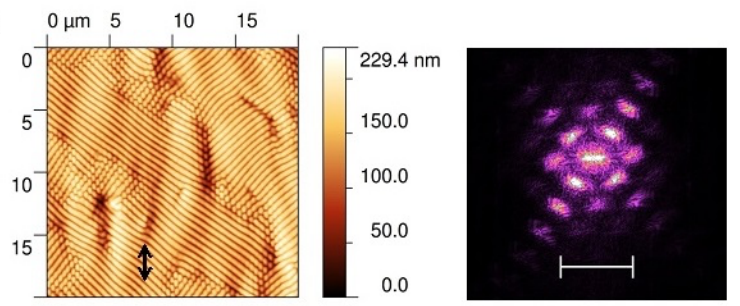

(b)

(c)
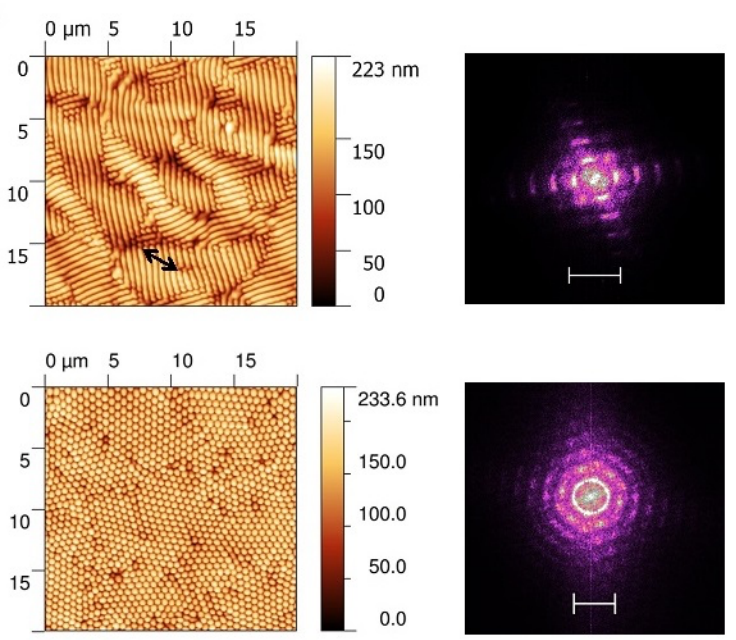

Fig. 3. AFM images and related Fourier transforms of the SSRGs printed with (a) vertical, (b) 150 degrees and (c) circular pump polarizations. Sample thickness was $270 \mathrm{~nm}$ and pump intensity was $300 \mathrm{~mW} / \mathrm{cm}^{2}$. Arrows indicate the polarization direction. Scale bars in the Fourier transforms are $5 \mu \mathrm{m}^{-1}$ long.

Irradiation of thin films of DR1-glass with $300 \mathrm{~mW} / \mathrm{cm}^{2}$ pump beam intensity resulted in SSRG formation under both linear and circular polarization, as evidenced by AFM (Fig. 3). This confirms that SSRG formation in azo-materials is an optical process, rather than a rheological one. As shown in Figures 3 a and b, which display the AFM scans and the associated Fourier transforms of SSRGs recorded with two different polarization directions, the direction of the gratings depends on the polarization direction of the pump beam. For linear polarization, the SSRGs consist of a patchwork of domains with gratings along two different directions oriented at + and $-40 \pm 5$ degrees relative to the pump beam polarization direction. A similar pattern was observed using DR1PMMA polymer [17]. The origin of orientation differences between SSRGs recorded in different materials is currently under study in our team. It may be attributed to the photoinduced anisotropy, which alters the direction into which mass transport happens [10]. The maximum modulation depth is $80 \pm 10 \mathrm{~nm}$ and the grating pitch is $480 \pm 30 \mathrm{~nm}$ for linear polarization. Circular polarization does not lead to any preferential self- organized grating direction, instead yielding a lattice of dome-like structures with two superimposed grating directions, with a grating pitch and depth 
after 1-hour irradiation ( $\left.422 \mathrm{~mW} / \mathrm{cm}^{2}\right)$ of $480 \pm 30 \mathrm{~nm}$ and $80 \pm 10$ $\mathrm{nm}$, respectively. For normal incident light, gratings with a pitch $\lambda / \mathrm{n}_{0}$ are expected to form according to the stimulated Wood anomaly theory [13], in which no is the effective refractive index of the medium above the surface. Practically, the medium above the surface refers to the selvedge region, with refractive index $\mathrm{n}_{0}=1.1$, in between the glass and air ones.

Importantly for applications in which holographic patterns are used as guides or templates [24], these gratings are optically reversible by inscribing a SSRG over an existing one with either linear (perpendicular to the initial polarization) or circular polarization. However, only partial thermal erasure was observed upon heating samples above their glass transition temperature $\left(71^{\circ} \mathrm{C}\right)$ during $30 \mathrm{~min}$ at $115^{\circ} \mathrm{C}$.

Fig. 4 shows the diffraction efficiency of the probe recorded as a function of time for identical samples irradiated with different pump intensities. Diffraction efficiency in the backward direction follows the same trend. The first- order forward diffracted light of SSRGs (at $46 \pm 2$ degrees) satisfies the total internal reflection condition at the glass substrate/air interface and at the DR1glass/air and it couples into the sample. As gratings are generated on the film surface, the diffraction efficiency of the probe increases. AFM has shown that SRGs reached their maximum amplitude in 10 min [20]. It takes about $20 \mathrm{~min}$ for SSRGs to reach their maximum amplitude in Fig. 4b, which shows that self-organization requires additional time to settle. We also see in Fig. 4a that diffraction efficiency continues to grow after 20 min irradiation, meanwhile the modulation amplitude reached its maximum. This may be attributed to photoinduced transparency, which takes place after relief formation (see below). For intensities below 25 $\pm 5 \mathrm{~mW} / \mathrm{cm}^{2}$ ordered SSRGs did not form, whereas intensities above $3 \mathrm{~W} / \mathrm{cm}^{2}$, caused ablation of the material. In this intensity range, the growth rate is proportional to the intensity, but the gratings saturate to the same amplitude.
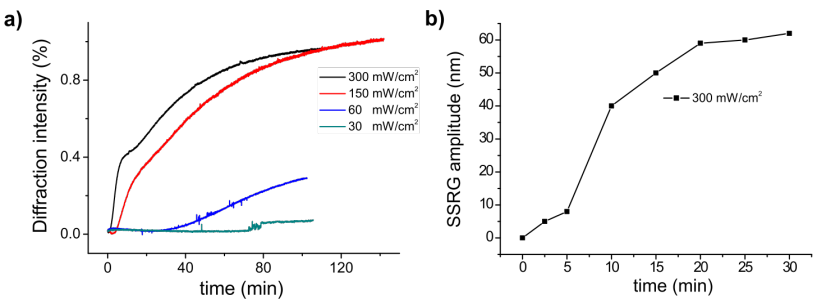

Fig. 4. (a) Diffraction efficiency as a function of time for different laser beam intensities. (b) SSRG modulation amplitude measured by AFM.

The irradiated area shows significant bleaching, which increases with irradiation time and laser intensity. It is understood that the cis isomers of azobenzenes can undergo reversible photocyclization to the colorless dihydrobenzocinnoline derivatives, which can be irreversibly trapped upon reaction with oxygen [25]. Study of thermal erasure reveals that the photoinduced bleaching is partially reversible thermally, but reversibility of the bleaching decreases with irradiation time, eventually yielding fully transparent gratings after $2 \mathrm{~h}$ exposure. The bleaching effect is not related to SSRG formation as it continues after SSRG amplitude saturation. However, this may explain why diffraction efficiency still increases after saturation of the modulation amplitude in Fig. 4.
As mentioned previously, first-order forward diffracted light can be coupled and trapped into the sample plane, with good control over the grating parameters and out-coupling conditions. The position of the out-coupled light varies with experimental parameters such as light polarization, thickness of the substrate, and diameter of the irradiated area. The first-order diffracted pump beam can be reflected onto the azo film in different locations and induce new periodic structures which could couple light out (Fig. 5a). This is evidenced by the observation of back-diffracted light (Fig. 5b). As a result, in addition to the irradiated area, four other faded areas outside the irradiated area are observed (Fig. $5 \mathrm{c}$ d), they are similar to ones observed by Hubert et al. [17]. By increasing the glass substrate thickness, the distance between these side areas relative to the central irradiated area also increases. AFM scans of the four side lobes reveal the existence of incoherent surface deformation, which indicates that the SSRG provides strong enough first order forward diffraction that the diffracted beams can induce mass transport of the azo material. However, these deformations do not construct coherent gratings in the absence of the incident beam (Fig. 6a) and look similar to dewetted patterns [26]. These observations further suggest that light coupling between the incident laser beam and scattered light at the sample is at the origin of SSRG formation, as modeled by Leblond et al. [27].

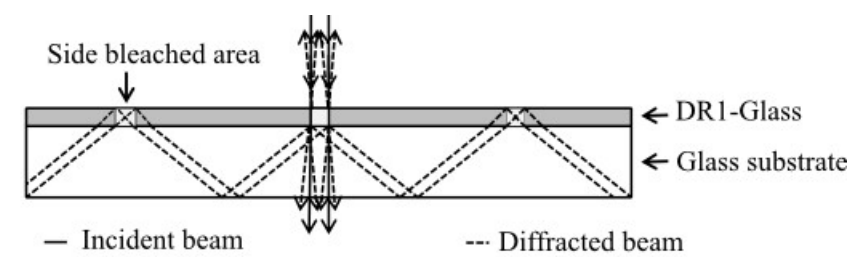

(a)

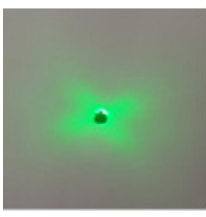

(b)

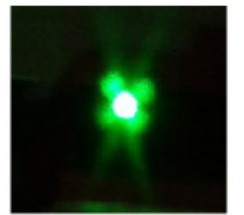

(c)

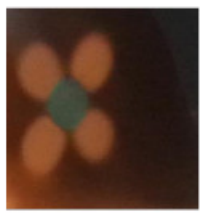

(d)
Fig. 5. a) Coupled light pathways in films of DR1-glass, b) back diffraction pattern, c) SSRG and side lobes under laser beam irradiation and d) in the absence of the laser.

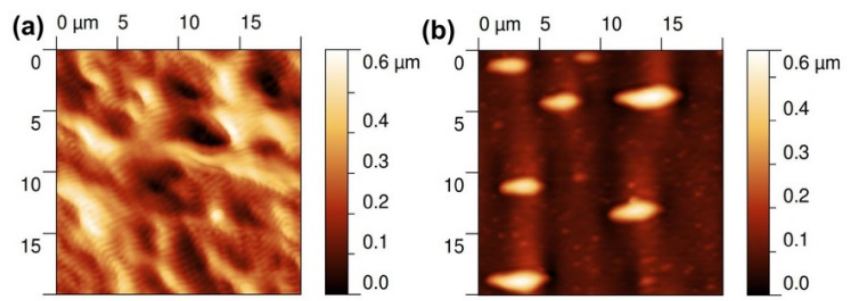

Fig. 6. AFM images of a) side lobes formed by light coupling, and b) ripples formed by irradiation from substrate side.

Irradiation from the substrate side also did not lead to coherent SSRG formation, as the process is surface-initiated. Ripples with a 3-5 $\mu \mathrm{m}$-pitch were formed in that situation (Fig. 6b). Dependence of this surface deformation on light polarization was investigated, 
and the wave vector of the ripples aligned perpendicular to the light polarization. This indicates that increased fluidity along the polarization direction is the main reason causing the ripples. The phenomenon cannot be attributed to photo thermal effects because under the laser intensities used, the temperature of the azo film would not increase enough to promote any significant photothermal effects to occur [28]. However, intensive photoisomerization and intermolecular interactions soften the matrix of the azo film, as it would happen under heating [29-32].

In conclusion, self-organized SRGs on a glass-forming DR1 derivative were investigated under uniform laser beam irradiation. SSRGs form according to the stimulated Wood anomaly theory. Light polarization is the main factor determining the orientation of the structures. The dependence of the grating growth rate on the intensity of the writing beam was studied. The rate of grating growth increases as a function of laser intensity, but the gratings saturate at the same modulation amplitude independently of the laser intensity. These gratings can be erased optically and thermally, and superimposed gratings with polarization multiplexing can be formed. The fabricated SSRGs provide a flexible way to couple light into the sample, leading to the formation of incoherent surface structures. Substrate side irradiation does not result in SSRG formation, instead yielding micron-level ripples due to photo-fluidity along the light polarization direction.

Funding: at Queen's - The National Science Research Council of Canada (NSERC) - Discovery Grant (RGPIN-2015-05485) and CREATE (Novel Chiral Materials: An International Effort in Research and Education).

\section{References}

1. J. Paterson, A. Natansohn, P. Rochon, C. L. Callender and L. Robitaille, Appl. Phys. Lett. 69, 3318 (1996)

2. T. Hattori, T. Shibata, S. Onodera and T. Kaino, Jap. J. App. Phys. 43, 1492 (2004)

3. E. Emoto, T. Uchida and T. Fukuda, Polymers 4, 150 (2012)

4. S. J. Zilker, T. Bieringer, D. Haarer, R. S. Stein, J. W. van Egmond and S. G. Kostromine, Adv. Mater. 10, 855 (1998)

5. C. Hubert, A. Rumyantseva, G. Lerondel, J. Grand, S. Kostcheev, L. Billot, A. Vial, R. Bachelot, and P. Royer, Nano Lett. 5, 615 (2005)

6. R. Bachelot, F. H'Dhili, D. Barchiesi, G. Lerondel, R. Fikri, P. Royer, N. Landraud, J. Peretti, F. Chaput, G. Lampel, J.-P. Boilot, and K. Lahlil, J. Appl. Phys. 94, 2060 (2003)

7. K. Li, H. Zhen, Zh. Huang, G. Li, and X. Liu, ACS Appl. Mater. Inter. 4, 4393 (2012)

8. C. Fiorini, N. Prudhomme, A. C. Etile, P. Lefin, P. Raimond, and J. M. Nunzi, Macromol. Symp. 137,105 (1999)

9. T. Todorov, L. Nikolova, and T. Tomova, Appl. Opt. 23, 4309 (1984).

10. P. Lefin, C. Fiorini and J. M. Nunzi, Opt. Mater.9, 323 (1998)

11. C.J. Barrett, A.L. Natansohn, P.L. Rochon, J. Chem. Phys. 100, 8836 (1996)

12. Z. Sekkat and W. Knoll, Photoreactive organic thin films, Academic Press, USA (2002)

13. A.E. Siegman and P.M. Fauchet, IEEE J. Quantum Electron. 22, 1384 (1986)

14. M. Siegrist, G. Kaech and F. K. Kneubühl, Appl. Phys. 2, 45 (1973)

15. N. Tsutsumi, and A. Fujihara, Appl. Phys. Lett. 85, 20 (2004)

16. S Ahmadi Kandjani, R Barille, S Dabos-Seignon, J. M. Nunzi, E Ortyl and S Kucharski, Opt. Lett. 30, 1986 (2005)

17. C Hubert, C Fiorini-Debuisschert, L Rocha, P Raimond, and J. M. Nunzi, J. Opt. Soc. Am. B 24,1839 (2007)
18. A. Ambrosio, S. Girardo, A. Camposeo, D. Pisignano, and P. Maddalena, Appl. Phys. Lett. 102, 093102 (2013)

19. J. M. Inytskyi, D. Neher, and M. Saphiannikova, J. Chem. Phys. 135, 14476 (2011)

20. R. Kirby, R. G. Sabat, J. M. Nunzi and O. Lebel, J. Mater. Chem C 2, 841 (2014)

21. H. Nakano, T. Takahashi, T. Kadota and Y. Shirota, Adv. Mater. 14, 1157 (2002)

22. E. Ishow, B. Lebon, Y. He, X. Wang, L. Bouteiller, L. Galmiche and K. Nakatani, Chem. Mater. 18, 1261 (2006).

23. K. Traskovskis, I. Mihailovs, A. Tokmakovs, A. Jurgis, V. Kokars and M. Rutkis, J. Mater. Chem. 22, 11268 (2012)

24. C. Rianna, A. Calabuig, M. Ventre, S. Cavalli, V. Pagliarulo, S Grilli, P. Ferraro, and P.A. Netti, ACS Appl. Mater. Interfaces 7, 16984 (2015)

25. J. Griffiths, Chem. Soc. Rev 1, 481 (1972)

26. E. Melito, A. Laventure, G. Aldea-Nunzi, C. Pellerin, E. Buncel, O. Lebel and J.M. Nunzi, J. Mater. Chem. C 3, 4729 (2015)

27. H. Leblond, R. Barille, S. Ahamadi-Kandjani, J.M. Nunzi, E. Ortyl and S. Kucharski, J. Phys B. 42, 205401 (2009)

28. K.G. Yager and C. J. Barrett, J. Chem. Phys. 120, 1089 (2004)

29. P. Karageorgiev, D. Neher, B. Schulz, B. Stiller, U. Pietsch, M. Giersig and L. Brehmer, Nature Materials 4, 699 (2005)

30. W. Li, T. Dohi, M. Hara, Sh. Nagano, O. Haba, K. Yonetake, and T. Seki, Macromol. 45, 6618 (2012)

31. J. Vapaavuori, A. Laventure, C. G. Bazuin, O. Lebel, and C. Pellerin, J. Am. Chem. Soc. 137,13510 (2015)

32. V. Teboul, M. Saiddine, and J.M. Nunzi, Phys. Rev. Lett. 103, 265701 (2009) 


\section{References}

1. J. Paterson, A. Natansohn, P. Rochon, C. L. Callender and L. Robitaille, "Optically inscribed surface relief diffraction gratings on azobenzene containing polymers for coupling light into slab waveguides" , Appl. Phys. Lett. 69, 3318-3320 (1996)

2. T. Hattori, T. Shibata, S. Onodera and T. Kaino, "Thermo-optically tunable wavelength filter with permanent refractive index grating into azopolymer waveguide", Japanese Journal of Applied Physics, 43, 14921495 (2004)

3. E. Emoto, T. Uchida and T. Fukuda, "Optical and physical applications of photocontrollable materials: azobenzene-containing and liquid crystalline polymers", Polymers 4, 150 (2012)

4. S. J. Zilker, T. Bieringer, D. Haarer, R. S. Stein, J. W. van Egmond, S. G. Kostromine, "Holographic data storage in amorphous polymers", Adv. Mater. 10, 855 (1998)

5. C. Hubert, A. Rumyantseva, G. Lerondel, J. Grand, S. Kostcheev, L. Billot, A. Vial, R. Bachelot, and P. Royer, "Near-Field Photochemical Imaging of Noble Metal Nanostructures", Nano Lett. 5, 615 (2005)

6. R. Bachelot, F. H'Dhili, D. Barchiesi, G. Lerondel, R. Fikri, P. Royer, N. Landraud, J. Peretti, F. Chaput, G. Lampel, J.-P. Boilot, and K. Lahlil, "Apertureless near-field optical microscopy: A study of the local tip field enhancement using photosensitive azobenzene-containing films" J. Appl. Phys. 94, 2060 (2003) ;

7. K. Li, H. Zhen, Zh. Huang, G. Li, and X. Liu, "Embedded surface relief gratings by a simple method to improve absorption and electrical properties of polymer solar cells", ACS Appl. Mater. Interfaces4 (8), 4393 (2012)

8. C. Fiorini, N. Prudhomme, A. C. Etile, Ph. Lefin, P. Raimond, and J. M. Nunzi, "All-optical manipulation of azo-dye molecules", Macromol. Sym. 137,105 (1999)

9. T. Todorov, L. Nikolova, and T. Tomova, "Polarization holography: a new high-efficiency organic material with reversible photoinduced birefringence," Appl. Opt. 23, 4309-4312 (1984).

10. Ph. Lefin, C. Fiorini, and J.M. Nunzi, "Anisotropy of the photoinduced translation diffusion of azo-dyes", Opt. Mater.9, 323 (1998)

11. C.J. Barrett, A.L. Natansohn, and P.L. Rochon, " Mechanism of optically inscribed high-efficiency diffraction gratings in azo polymer films", J. Chem. Phys., 100, 8836-8842 (1996)

12. Z. Sekkat, W. Knoll, "Photoreactive organic thin films", Academic Press, USA (2002)

13. A.E. Siegman, and P.M. Fauchet, "stimulated Wood's Anomalies on Laser-Illuminated Surfaces”, IEEE J. Quantum Electron. 22, 1384-1403 (1986)

14. M. Siegrist, G. Kaech, and F. K. Kneubühl, "Formation of a periodic wave structure on the dry surface of a solid by TEA-CO2-laser pulses", Appl. Phys. 2, 45-46 (1973)

15. N. Tsutsumi and A. Fujihara, "Pulsed laser induced spontaneous gratings on a surface of azobenzene polymer" Appl. Phys. Lett. 85, 20 (2004)

16. S Ahmadi Kandjani, R Barille, S Dabos-Seignon, J-M Nunzi, E Ortyl, and S Kucharski, "Multistate polarization addressing using a single beam in an azo polymer film", Opt. Lett. 30, 1986 (2005)

17. C Hubert, C Fiorini-Debuisschert, L Rocha, P Raimond, and J.M.Nunzi, "Spontaneous photoinduced patterning of azo-dye polymer films: the facts", J. Opt. Soc. Am. B 24(8), 1839-1846 (2007)

18. Ambrosio, S. Girardo, A. Camposeo, D. Pisignano, and P. Maddalena, "Controlling spontaneous surface structuring of azobenzene-containing polymers for large-scale nano-lithography of functional substrates", Appl. Phys. Lett. 102, 093102 (2013)

19. J. M. Ilnytskyi, D. Neher, and M. Saphiannikova. "Opposite photoinduced deformations in azobenzene-containing polymers with different molecular architecture: molecular dynamics study.", J. Chem. Phys. 135, 14476 (2011)
20. R. Kirby, R. G. Sabat, J. M. Nunzi, and O. Lebel , "Disperse and disordered: a mexylaminotriazine-substituted azobenzene derivative with superior glass and surface relief grating formation." J. Mater. Chem C 2, 841-847 (2014)

21. H. Nakano, T. Takahashi, T. Kadota and Y. Shirota "Formation of a Surface Relief Grating Using a Novel Azobenzene-Based Photochromic Amorphous Molecular Material."Adv. Mater. 14 1157-1160 (2002)

22. E. Ishow, B. Lebon, Y. He, X. Wang, L. Bouteiller, L. Galmiche and K. Nakatani . "Structural and photoisomerization cross studies of polar photochromic monomeric glasses forming surface relief gratings." Chem. Mater. 18, 1261-1267 (2006)

23. K. Traskovskis, I. Mihailovs, A. Tokmakovs, A. Jurgis, V. Kokars and M. Rutkis. "Triphenyl moieties as building blocks for obtaining molecular glasses with nonlinear optical activity." J. Mater. Chem. 22, 11268-11276 (2012)

24. C. Rianna, A. Calabuig, M. Ventre, S. Cavalli, V. Pagliarulo, S Grilli, P. Ferraro, and P.A. Netti, "Reversible Holographic Patterns on Azopolymers for Guiding Cell Adhesion and Orientation" ACS Appl. Mater. Interfaces 7, 16984-16991 (2015)

25. J. Griffiths, "Photochemistry of azobenzene and its derivatives" Chem. Soc. Rev. 1, 481-493 (1972)

26. E. Melito, A. Laventure, G. Aldea-Nunzi, C. Pellerin, E. Buncel, O. Lebel, and J.M. Nunzi, «Water-triggered spontaneous surface patterning in thin films of mexylaminotriazine molecular glasses», J. Mater. Chem. C 3, 4729 (2015)

27. H. Leblond, R. Barille, S. Ahamadi-Kandjani, J.M. Nunzi, E. Ortyl and S. Kucharski, 'Spontaneous formation of optically induced surface relief gratings', J. Phys B. 42, 205401 (2009)

28. K.G. Yager and Ch. J. Barrett, "Temperature modeling of laser-irradiated azo-polymer thin films" J. Chem. Phys. 120, 1089 (2004)

29. P. Karageorgiev, D. Neher, B. Schulz, B. Stiller, U. Pietsch, M. Giersig and L. Brehmer, "From anisotropic photo-fluidity towards nanomanipulation in the optical near-field", Nature materials 4,699 (2005)

30. W. Li, T. Dohi, M. Hara, Sh. Nagano, O. Haba, K. Yonetake, and T. Seki, "Phototriggered mass migration consorted with surface dewetting in thin films of a liquid crystalline azobenzene-containing dendrimer", Macromol. 45, 6618 (2012)

31. J. Vapaavuori, A. Laventure, C. G. Bazuin, O. Lebel, and Ch. Pellerin, "Submolecular Plasticization Induced by Photons in Azobenzene Materials." J. Am. Chem. Soc. 137,13510-13517 (2015)

32. V. Teboul, M. Saiddine, and J.M. Nunzi, 'An isomerization-induced dynamic heterogeneity in a supercooled glass-former', Phys. Rev. Lett. 103, 265701 (2009) 